\title{
Impact of Rectal Swabs on Infectious Complications after Transrectal Prostate Biopsy
}

\author{
Carlos Gustavo Trujillo ${ }^{a}$ Mauricio Plata ${ }^{a}$ Juan Ignacio Caicedo ${ }^{a}$ \\ Juan Guillermo Cataño Cataño ${ }^{b}$ Angela Marcela Mariño Alvarez ${ }^{a}$ \\ Diana Castelblanco ${ }^{\text {b }}$ Daniela Robledo ${ }^{\text {a }}$
}

${ }^{a}$ Department of Urology, Hospital Universitario de la Fundación Santa Fe de Bogotá and Universidad de los Andes School of Medicine, and bepartment of Urology, Hospital Universitario de la Fundación Santa Fe de Bogotá,

Bogotá, D.C., Colombia

\section{Key Words}

Prostate · Prostate cancer · Prostate biopsy · Complication · Infection · Antibiotic prophylaxis

\begin{abstract}
Objectives: To determine the impact of rectal swabs (RSs) on infectious complications (IC) following prostate biopsy (PB). Methods: A retrospective cohort study was conducted including all patients subjected to PB between 2009 and 2013. Group B consisted of patients with a RS and group A of patients without. RS reported the presence of gram-positive or negative germs, sensitive or resistant to ciprofloxacin. Antimicrobial prophylaxis was adjusted to the result. Frequency of IC in each group was determined. Results: Group B had 548 (47.20\%) patients and group A 613 (52.80\%). From group B, $250(45.62 \%)$ of the RSs showed fluoroquinolone (FQ)-resistant germs. Forty nine (16.44\%) patients with sensitive germs vs. 147 (59.51\%) with resistant germs had a history of previous FQ treatment $(p<0.0001)$. IC were observed in 33 (5.49\%) patients from group $A$ and in $7(1.28 \%)$ patients from group B ( $p<0.0001)$, requiring hospitalization in 4.99 vs. $1.28 \%$, respectively. IC and hospital admissions were reduced in 76.68 and $74.34 \%$, respectively, following the im-
\end{abstract}

plementation of RS. Conclusions: RS and targeted antibiotic prophylaxis prior to PB was associated with a significant reduction in IC and hospital admissions. Ceftriaxone could be an alternative in cases of known resistance. Past history of FQ treatment is associated with increased resistance.

(C) 2016 S. Karger AG, Base

\section{Introduction}

Transrectal ultrasound (TRUS)-guided prostate biopsy $(\mathrm{PB})$ is one of the urological procedures more frequently performed, with a yearly estimate of over 1,000,000 in the United States [1]. Although it is usually done on an outpatient basis with a low rate of complications, infectious events such as urinary tract infections (UTI), prostatitis, bacteremia, and sepsis are among the most feared [2]. Gram-negative bacilli are the most frequently isolated germs, particularly Escherichia coli.

The benefit of prophylactic antibiotic therapy in TRUS-guided PB has been reported in placebo-controlled studies, with a reduction in infectious complications (IC) ranging between 8 and 25\% [3]. A Cochrane meta-analysis showed that the number of patients with

\section{KARGER}

E-Mail karger@karger.com

www.karger.com/uin (c) 2016 S. Karger AG, Base

0042-1138/16/0973-0340\$39.50/0
Carlos Gustavo Trujillo, MD

Department of Urology, Fundación Santa Fe de Bogotá University Hospital and Universidad de los Andes School of Medicine, Carrera 7 No. 118-09, Unidad Renal Piso 3, Clínicas Urológicas de la Fundación Santa Fe de Bogotá, Bogotá DC 110111 (Colombia), E-Mail cg.trujillo2592@ uniandes.edu.co 
bacteriuria, bacteremia, fever, and the need for hospitalization decreased with the use of antimicrobial prophylaxis [4]. Fluoroquinolones (FQs) are the gold standard for prophylaxis, mainly due to properties such as a good penetration of the prostatic tissue. However, a growing resistance to FQ has led to a 4-fold increase in the number of post-biopsy episodes of infection [5], which in turn has put to question their prophylactic usefulness.

To decrease the rate of IC and to avoid the use of FQ in resistant cases, we implemented a rectal swab (RS)/culture and targeted prophylactic antibiotic therapy protocol before TRUS-guided PB in our institution. The purpose of this study was to determine the rate of IC in a group of patients with targeted antibiotic prophylaxis prior to $\mathrm{PB}$, compared to cases with non-targeted therapy.

\section{Materials and Methods}

A retrospective cohort study was conducted. All male patients who underwent PB in our institution (fourth-level, located in Colombia) between February 2009 and July 2013 were included in the study. The cohort was divided into 2 groups: group B consisted of patients with a RS and group A consisted of patients without. Within group A, the prophylactic antimicrobial schemes varied over time and were based on recommendations of clinical guidelines, our institution resistance profile, and the incidence of infectious adverse events. These schemes included ciprofloxacin, ciprofloxacin + metronidazole, ceftriaxone, amikacin, amikacin + ciprofloxacin, amikacin + metronidazole, cefuroxime, and metronidazole.

RS was performed one week prior to $\mathrm{PB}$ with the following protocol. Trained nurses introduce a cotton swab about one centimeter long through the anus and place the sample in Amies or Stuart medium, and send it to our clinical laboratory. The sample is then placed in Todd-Hewitt broth and incubated for $24 \mathrm{~h}$ at $35 \pm 2{ }^{\circ} \mathrm{C}$. A $0.5 \mathrm{McF}$ arland scale with saline $0-85 \%$ is prepared prior to inoculation in Mueller-Hinton agar, and a ciprofloxacin disk $(5 \mu \mathrm{g})$ is added. The sample is further incubated $\left(24 \mathrm{~h}\right.$ at $\left.35 \pm 2{ }^{\circ} \mathrm{C}\right)$, and inhibition halos are assessed after $24 \mathrm{~h}$. Resistance is determined and reported based on the Clinical and Laboratory Standards Institute M100 document (sensitive $>21 \mathrm{~mm}$, intermediate 16-20 $\mathrm{mm}$, resistant $<15 \mathrm{~mm}$ ). No samples were taken immediately after performing digital rectal examination since lubricants would have altered the results.

Rectal culture reported the presence of germs sensitive or resistant to ciprofloxacin, but not specific bacteria, given that the purpose of the study was to identify FQ resistance to select prophylaxis. When sensitive, a dose of $500 \mathrm{mg}$ ciprofloxacin was administered orally $1 \mathrm{~h}$ before the procedure, followed by a dose every $12 \mathrm{~h}$ for 5 days If the resistance was documented, a single dose of ceftriaxone ( $1 \mathrm{~g}$ ) was administered intravenously $1 \mathrm{~h}$ before the biopsy (both regimens were assigned by our institution's Committee for Infectious Diseases). All patients subjected to PB were considered free of active infections and were checked for a negative urine analysis and/or culture prior to the procedure. Semen cultures and Stamey tests were not performed since active bacterial prostatitis cases (confirmed or suspected) were excluded from biopsy, treated and rescheduled.

Two rectal enemas (16 g monobasic sodium phosphate USP and $6 \mathrm{~g}$ dibasic sodium phosphate USP $\left(\operatorname{Travad}^{\circledR}\right)$ ) were administered $2 \mathrm{~h}$ prior to the procedure. Biopsies were performed under local anesthesia ( $10 \mathrm{ml}$ of $2 \%$ xylocaine without epinephrine) with a transrectal approach by trained urologists and radiologists. At least 12 samples were collected per biopsy, corresponding to 2 samples per sextant.

A data collection format was constructed in which the use of antibiotics (particularly FQ) during the 6 months prior to the biopsy was registered. Following the procedure, all patients were monitored for the first month through telephonic interview or in person, and they were questioned on the occurrence of fever, urinary symptoms, and hospitalizations. When any of the answers were positive, medical records were reviewed for variables including vital signs, complete blood count, urinalysis, blood and urine cultures, and the need for hospital or intensive care unit admission. Patients who were admitted to the emergency room with a possible IC and urinary symptoms had urine cultures performed and were treated with antibiotics. Blood cultures were performed when patients developed a fever while being hospitalized. IC were defined based on the Surviving Sepsis Campaign Guidelines, 2013 [6] and the Global Prevalence Study of Infections in Urology 2010 and 2011 [5] (online suppl. Appendix 1, see www.karger.com/doi/10.1159/000448595) and patients were categorized according to the most severe complication recorded. All the information gathered was noted into a digital database.

Statistical analysis was conducted for measures of central tendency and dispersion using STATA 12.0. The frequency of events was recorded as proportions for categorical variables and averages for continuous variables. Normality in distribution was determined and parametric statistics were used to determine the interference between them.

\section{Results}

A total of 1,161 patients were included. Fourteen (1.19\%) subjects were excluded due to incomplete information or non-compliance to the protocol. Group A had 613 (52.80\%) patients and group B had 548 (47.20\%). Table 1 summarizes the baseline characteristics of each group. No difference was found between patients with a past history of $\mathrm{PB}$ and those without, with respect to having a FQ-resistant germ of the RS $(\mathrm{p}=0,681)$ or developing an IC $(\mathrm{p}=1)$.

RSs and cultures (group B) resulted in FQ-sensitive germs in $298(54.38 \%)$ of the cases and FQ-resistant germs in 250 (45.62\%). A history of past treatment with quinolones was present in $49(16.44 \%)$ patients with a FQ-sensitive germ vs. 147 (58.8\%) patients with a FQresistant germ (OR 7.47, 95\% CI 5.01-11.12, p < 0.0001) 


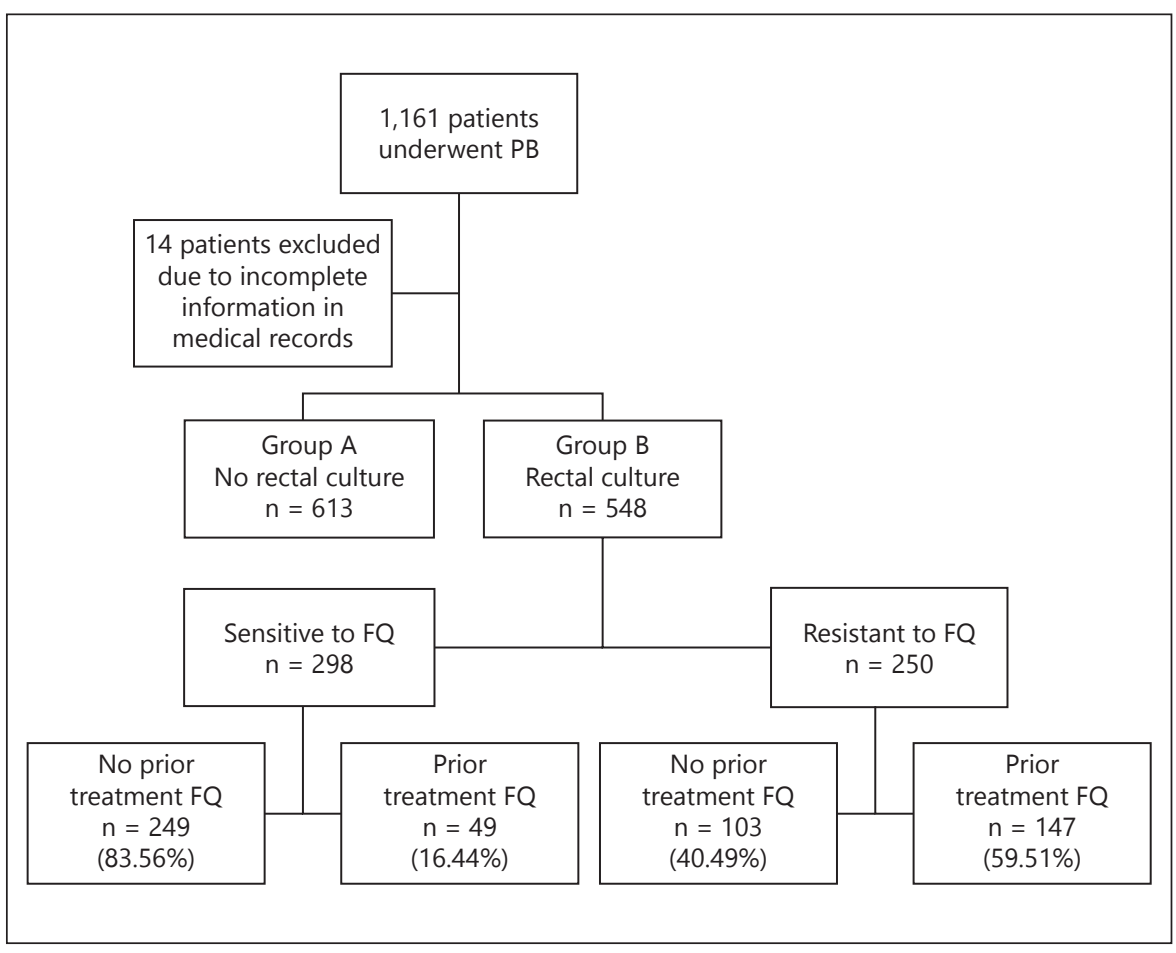

Fig. 1. Study profile.

Table 1. Baseline characteristics of groups A and B

\begin{tabular}{lcc}
\hline Variable & Group A (n=613) & Group B (n= 548) \\
\hline Age, years, median (range) & $62(39-97)$ & $62.4(33-92)$ \\
Prostate-specific antigen, ng/ml, median (range) & $7.72(0.36-258)$ & $7.00(0.30-112)$ \\
Past history of prostate biopsy, n (\%) & $50(18.66)^{\dagger}$ & $142(26.79)^{\boldsymbol{\top}}$ \\
Past history of FQ treatment, $\mathrm{n}(\%)$ & $4(1.29)^{\dagger \dagger}$ & $196(35.96)^{\S}$ \\
\hline
\end{tabular}

${ }^{\dagger} \mathrm{n}=268$ due to missing data.

${ }^{\dagger \dagger} \mathrm{n}=309$ due to missing data.

I $\mathrm{n}=530$ due to missing data.

$\S_{\mathrm{n}}=545$ due to missing data.

Table 2. IC discriminated by groups A and B

\begin{tabular}{lcl}
\hline Infectious complication & $\begin{array}{l}\text { Group A } \\
(\mathrm{n}=613), \%\end{array}$ & $\begin{array}{l}\text { Group B } \\
(\mathrm{n}=548), \%\end{array}$ \\
\hline Sepsis & $23(3.83)$ & $6(1.10)$ \\
Bacteremia & $3(0.50)$ & $0(0)$ \\
Urinary tract infection & $3(0.50)$ & $0(0)$ \\
Isolated fever & $2(0.33)$ & $0(0)$ \\
Prostatitis & $1(0.17)$ & $0(0)$ \\
Epididymitis & $1(0.17)$ & $1(0.18)$ \\
\hline
\end{tabular}

(fig. 1). A lack of exposure to FQ was found to be a protective factor for IC (OR 0.223, 95\% CI 0.097-0.508, p < 0.0001 ) conferring a risk reduction of $87.7 \%$ (49.2-90.3\%).

Forty patients from the total sample presented with IC, 33 (5.49\%) of which belonged to group A and 7 (1.28\%) to group B; this difference was statistically significant $(\mathrm{p}=$ 0.0001 ) (table 2). Urine culture was performed in $36 \mathrm{pa}-$ tients that required in-hospital management $(3.13 \%$ of the entire cohort); 29 belonged to group A and 7 to group B. Urine culture results and isolated germs are summarized in table 3 . Blood culture was performed in 23 of the hospitalized patients; only $4(17.39 \%)$ had bacterial 
Table 3. Isolated germs in urine cultures from patients with IC following transrectal PB

\begin{tabular}{lll}
\hline Variable & Group A $(\mathrm{n}=29)$ & Group B $(\mathrm{n}=7)$ \\
\hline Positive urine culture, $\mathrm{n}(\%)^{*}$ & $18(81.82)$ & $4(18.18)$ \\
Fluoroquinolone resistance, $\mathrm{n}(\%)$ & $4(80.00)^{\dagger}$ & $1(20.00)$ \\
\hline FQ-resistant isolated germs & E. coli $(\mathrm{n}=2)$ & E. coli $(\mathrm{n}=1)$ \\
& E. coli ESBL-producing $(\mathrm{n}=1)$ & \\
& Staphylococcus haemolyticus $(\mathrm{n}=1)$ & E. coli $(\mathrm{n}=1)$ \\
& E. coli $(\mathrm{n}=11)$ & Pseudomonas aeruginosa $(\mathrm{n}=1)$ \\
& Streptococcus agalactiae & coli + Klebsiella pneumoniae $(\mathrm{n}=1)$ \\
& E. coli + S. agalactiae $(\mathrm{n}=1)$ & \\
\hline
\end{tabular}

\footnotetext{
* No germ was isolated in 14 urine cultures (11 from group A and 3 from group B).

† Three of these patients had prophylaxis with ciprofloxacin $500 \mathrm{mg}$ PO BID for 5 days and one patient received 2 doses of amikacin $500 \mathrm{mg}$ IM BID $\times 1$ day plus metronidazole $500 \mathrm{mg}$ PO TID $\times 3$ days.
}

Table 4. Summary of findings in patients from group B with IC

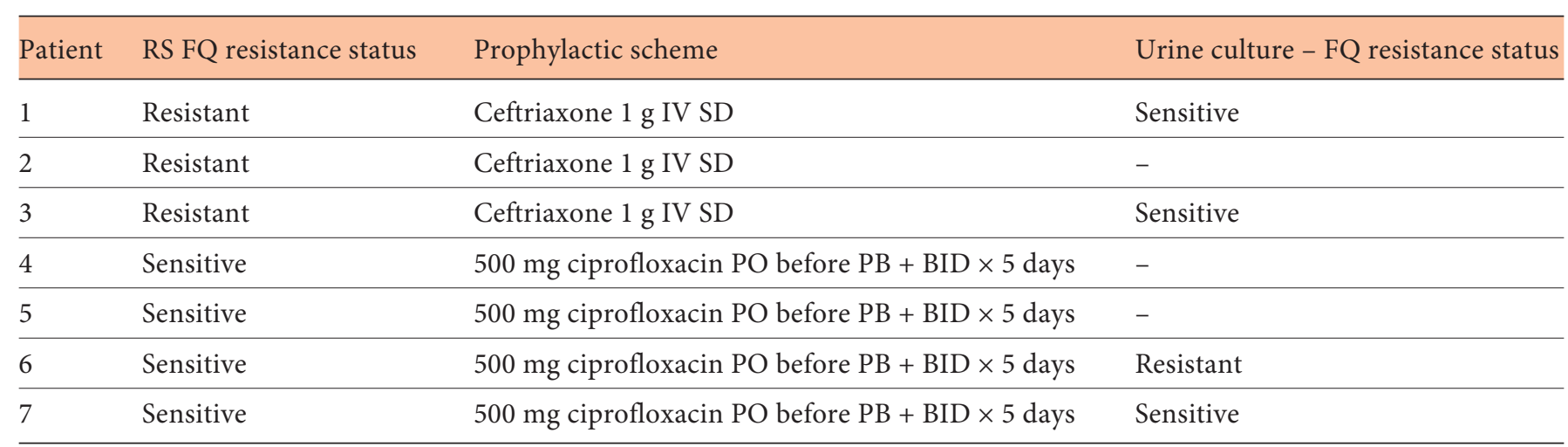

growth and 3 had the same germ as in the urine culture. All of these patients belonged to group A. Out of the 7 patients belonging to group B who developed an IC, 3 $(42.86 \%)$ had had a resistant germ in the RS and had received prophylaxis with ceftriaxone accordingly. Urine culture results in 2 of these patients were positive for FQsensitive germs (table 4).

Hospitalization was indicated in $4.99 \%$ of those belonging to group A compared to $1.28 \%$ of group B, which represents a decrease of IC and hospital admissions of 76.68 and $74.34 \%$, respectively (OR 0.246 , 95\% CI $0.11-$ $0.56, \mathrm{p}<0.001)$. The most common IC in both groups was sepsis. Table 3 shows the frequency of IC per group. There was no mortality in either group.

Impact of RSs on Infections Post Biopsy

\section{Discussion}

FQs currently constitute one of the most widely used type of antibiotics for PB prophylaxis due to its ability to penetrate prostatic tissue. A recent multicenter study reported that $92.5 \%$ of the patients that underwent $\mathrm{PB}$ received FQ [5]. However, the duration of therapy is different in most studies $[7,8]$.

Despite prophylaxis, IC remain the most dreaded among physicians [9]. Asymptomatic bacteriuria, UTI, and epididymitis are the most frequent IC, but more severe cases such as meningitis, vertebral osteomyelitis, sepsis, and septic shock have also been reported [10]. However, their frequency varies considerably. Simsir et 
al. [11] reported a rate of sepsis of 3.06\% in a sample of 2,023 patients in Turkey. De Jesus et al. [12] reported a $14.4 \%$ rate of post-PB IC consisting of $1.7 \%$ sepsis, $9.2 \%$ UTI, $0.6 \%$ orchitis, and $0.6 \%$ prostatitis. A nationwide survey conducted in Japan that included the information from 212,065 PB performed between 2004 and 2006, reported a $2.22 \%$ rate of IC, with epididymitis occurring in $0.06 \%$, prostatitis in $0.9 \%$, UTI in $0.09 \%$, and sepsis in $0.07 \%$ of the cases [13]. In a North American cohort of $1,446 \mathrm{~PB}$ patients recruited between 2001 and 2010, 2.77\% had IC of which $2.14 \%$ were UTIs and $0.62 \%$ developed sepsis [14]. In our institution during 2009, we found a $7 \%$ rate of post-PB IC [15] and Agresott-Guerra et al. [16] found a $4.3 \%$ rate of IC in a population similar to this study.

Most studies show a progressive increase in post-PB IC that is possibly due to the growing antibiotic resistance $[16,17]$. Nam et al. [18] conducted a study with 75,190 patients who underwent $\mathrm{PB}$ and reported a significant increase in the rate of infection-related hospital admission from $1 \%$ in 1996 to $4.1 \%$ in 2005 . Hospital admittance due to IC ranges between 0.62 and $6.3 \%[14,19,20]$. Carignan et al. [2] conducted a case-and-control study in Canada with a sample of 5,798 patients, and showed an increasing incidence of IC from 0.42 per 100 biopsies during 20022009 up to 2.15 per 100 biopsies during 2010-2011. Of the 48 IC cases, they reported $42 \%$ had bacteremia and $66.7 \%$ of the patients required hospitalization.

Increasing resistance to FQ antibiotics is a plausible explanation for the occurrence of these data. In our cohort, antibiotic resistance was reported in $45.62 \%$ of the cases and previous use of FQ in $58.8 \%$ of the patients, suggesting a strong relationship between these 2 findings. In a previous study of $141 \mathrm{~PB}$ carried out in Thailand, Siriboon et al. [21] found an association between previous antibiotic use and antimicrobial resistance, notably ESBL-production (22.2 vs. $40.7 \%)$ and ceftriaxone resistance (28.2 vs. $48.1 \%$ ). Furthermore, they found that ciprofloxacin resistance was significantly higher in patients who received antibiotic prophylaxis (adjusted OR 11.52, 95\% CI 1.65-80.6, $\mathrm{p}=0.014$ ) compared to those who did not.

Several strategies have been deployed in an effort to reduce the rate of IC and its economic impact. Rectal antisepsis with povidone-iodine prior to the procedure has shown variable results, though a reduction in the rate of epididymitis is evident in some studies [22, 23]. Another alternative is the use of enemas. A Cochrane review concluded that the use of enema with antibiotics reduced the risk of bacteremia compared to the use of antibiotics alone (RR 0.25, 95\% CI 0.08-0.75), but there were no differences for fever or infection [4]. Changing antibiotic prophylaxis schemes is yet another strategy. Some studies report decreased infections when adding ciprofloxacin to schemes of amoxicillin-clavulanate [24]. Conversely, replacing ciprofloxacin by amoxicillin-clavulanate and gentamicin increased the frequency of infections [25]. Another study showed that mixing $1 \mathrm{~g}$ of ceftriaxone with the periprostatic lidocaine injection was associated with a lower rate of sepsis [26]. Furthermore, other authors suggest that the length of the prophylaxis course may also play an important role, since prolonging prophylaxis beyond 24-48 h may increase the emergence of resistant bacterial strains [27]. In our study, prophylaxis with oral ciprofloxacin was continued for 5 days after the procedure.

Performing rectal cultures prior to $\mathrm{PB}$ to target antimicrobial prophylaxis seems to have a favorable effect, but this has been assessed mainly through non-randomized studies. One study administered ciprofloxacin to all patients except those who showed resistance to quinolones. Those patients who showed resistance to quinolones underwent targeted therapy according to the antimicrobial resistance profile, reporting an absence of IC [28]. Taylor et al. [29] showed a non-statistically significant reduction in the frequency of sepsis using targeted prophylaxis with rectal culture versus standard prophylaxis ( 0 vs. $2.6 \%, \mathrm{p}=0.12$ ). Liss et al. [30] reported similar findings in a retrospective analysis of 5,355 prostate biopsies. The incidence of post-PB sepsis between the empirical versus targeted prophylaxis group was not significantly different (0.56 vs. $0.44 \%, p=0.568)$, however, targeted therapy prevented the use of more than one broad-spectrum empirical antibiotic.

The rate of FQ-resistance in patients with pre-PB rectal culture ranges between 3.7 and 25\% [30-33]. In our study, FQ-resistance was $45.62 \%$. It is also notable that $41.2 \%$ of the patients with no past history of quinolone use showed resistance to this antibiotic. The indiscriminate use of antibiotics for the treatment of urinary tract disorders and other conditions, and the use of antibiotics in food products may be factors contributing to resistance [34].

Steensels et al. [35] carried out a prospective study in which RSs were performed immediately prior to the biopsy. Of 236 cultures, 178 (75\%) had FQ-sensitive strains of $E$. coli, which differs greatly from our $54 \%$. In the remaining 58 cases, 52 had FQ-resistant strains of $E$. coli and the other 6 grew Pseudomonas aeruginosa $(\mathrm{n}=2)$, 
Comamonas kerstersii $(\mathrm{n}=2)$, Candida albicans, and Proteus mirabilis. Overall, $22 \%$ of the patients were found to have a FQ-resistant germ. Similar to our study, the main risk factor for having a FQ-resistant strain was the use of FQ during the 6 months prior to the biopsy $(\mathrm{p}<0.01)$, and repeat biopsy was not found to be a risk factor. Only $7 \%$ of the patients had IC, all of them caused by ciprofloxacin-resistant strains and 6 patients (2.5\%) developed sepsis, 5 of them with resistant strains.

In a systematic review of 9 studies with 4,571 patients by Cussans et al. [36], 45\% (2807) had pre-biopsy RSs. FQ-resistance was found on an average in $23 \%$ of the patients. IC and sepsis were more likely to happen with non-targeted prophylaxis. Absolute risk reduction was 3.83\% (95\% CI 2.94-4.93), and based on their findings 27 men would need to receive targeted antimicrobial therapy to prevent one case of IC (95\% CI $21.2-$ 34.1).

Our study documented a statistically significant reduction in the number of IC and hospital admissions, with the implementation of a pre-PB rectal culture protocol. Additionally, we had a rate of $17.39 \%$ positive blood cultures, which is lower in comparison to other series. Limitations of this study include its retrospective and non-randomized design, and that no risk factors (such as previous urinary sepsis, bacterial prostatitis, organ trans- plantation and immunosuppression) were assessed. Identifying specific bacteria rather than just FQ-resistance might be helpful in further reducing IC. However, our results are sufficient evidence for us to implement RSs as a strategy to lower the rate of IC.

\section{Conclusions}

Performing a RS and culture prior to $\mathrm{PB}$ and targeting antimicrobial prophylactic therapy accordingly, resulted in a statistically significant decrease in IC and the need for hospitalization in our cohort. We consider that this approach should be considered as a standard of care. Although FQ are still the drug of choice, in cases of known resistance a single dose of ceftriaxone might be a safe alternative. In our country, resistance to FQ is high and past history of FQ use appears to be a significant risk factor, but randomized prospective studies are needed to confirm this finding.

\section{Disclosure Statement}

No external sources of financial or material support were received for this work.

\section{References}

$\checkmark 1$ Loeb S, Vellekoop A, Ahmed HU, Catto J, Emberton M, Nam R, et al: Systematic review of complications of prostate biopsy. Eur Urol 2013;64:876-892.

-2 Carignan A, Roussy JF, Lapointe V, Valiquette L, Sabbagh R, Pépin J: Increasing risk of infectious complications after transrectal ultrasound-guided prostate biopsies: time to reassess antimicrobial prophylaxis? Eur Urol 2012;62:453-459.

- 3 Aron M, Rajeev TP, Gupta NP: Antibiotic prophylaxis for transrectal needle biopsy of the prostate: a randomized controlled study. BJU Int 2000;85:682-685.

4 Zani EL, Clark OA, Rodrigues Netto N Jr: Antibiotic prophylaxis for transrectal prostate biopsy. Cochrane Database Syst Rev 2011; 5:CD006576.

5 Wagenlehner FM, van Oostrum E, Tenke P, Tandogdu Z, Çek M, Grabe M, et al: Infective complications after prostate biopsy: outcome of the global prevalence study of infections in urology (GPIU) 2010 and 2011, a prospective multinational multicentre prostate biopsy study. Eur Urol 2013;63:521-527.

-6 Dellinger RP, Levy MM, Rhodes A, Annane D, Gerlach H, Opal SM, et al: Surviving sepsis campaign: international guidelines for management of severe sepsis and septic shock: 2012. Crit Care Med 2013;41:580-637.

7 Smyth LG, Mulvin DW: Antibiotic prophylaxis for transrectal ultrasound biopsy of the prostate in Ireland. Ir J Med Sci 2012;181:3335.

8 Briffaux R, Coloby P, Bruyere F, Ouaki F, Pires C, Doré B, Irani J: One preoperative dose randomized against 3-day antibiotic prophylaxis for transrectal ultrasonographyguided prostate biopsy. BJU Int 2009;103: 1069-1073; discussion 1073.

-9 Bootsma AM, Laguna Pes MP, Geerlings SE, Goossens A: Antibiotic prophylaxis in urologic procedures: a systematic review. Eur Urol 2008;54:1270-1286.

10 Carmignani L, Picozzi S, Spinelli M, Di Pierro S, Mombelli G, Negri E, et al: Bacterial sepsis following prostatic biopsy. Int Urol Nephrol 2012;44:1055-1063.

11 Simsir A, Kismali E, Mammadov R, Gunaydin G, Cal C: Is it possible to predict sepsis, the most serious complication in prostate biopsy? Urol Int 2010;84:395-399.

12 de Jesus CM, Corrêa LA, Padovani CR: Complications and risk factors in transrectal ultra- sound-guided prostate biopsies. Sao Paulo Med J 2006;124:198-202.

13 Kakehi Y, Naito S; Japanese Urological Association: Complication rates of ultrasoundguided prostate biopsy: a nation-wide survey in Japan. Int J Urol 2008;15:319-321.

14 Zaytoun OM, Vargo EH, Rajan R, Berglund $\mathrm{R}$, Gordon S, Jones JS: Emergence of fluoroquinolone-resistant Escherichia coli as cause of postprostate biopsy infection: implications for prophylaxis and treatment. Urology 2011; 77:1035-1041.

15 Castelblanco D, Jaramillo FG, Ordoñez CGT: Análisis retrospectivo de las biopsias de próstata realizadas en la Clínica de Próstata del Hospital universitario Fundación santa $\mathrm{Fe}$ de Bogotá. Urol Colomb 2011;20:27-33.

16 Agresott-Guerra WA, Shek-Padilla ÁH, Quiroga-Matamoros W, Citarella-Otero DA: Biopsia transrectal de próstata guiada por ecografía con 12 muestras de la zona periférica y muestras de la zona centro transicional en rebiopsias para el diagnóstico de cáncer de próstata. Revista Urología Colombiana (Internet). 2011. http://www.redalyc.org/ articulo.oa?id=149122333004 (February 11, 2016). 
$\checkmark 17$ Dodds PR, Boucher JD, Shield DE, Bernie JE, Batter SJ, Serels SR, et al: Are complications of transrectal ultrasound-guided biopsies of the prostate gland increasing? Conn Med 2011; 75:453-457.

18 Nam RK, Saskin R, Lee Y, Liu Y, Law C, Klotz $\mathrm{LH}$, et al: Increasing hospital admission rates for urological complications after transrectal ultrasound guided prostate biopsy. J Urol 2013;189(1 suppl):S12-S17; discussion S17S18.

19 Feliciano J, Teper E, Ferrandino M, Macchia RJ, Blank W, Grunberger I, Colon I: The incidence of fluoroquinolone resistant infections after prostate biopsy - are fluoroquinolones still effective prophylaxis? J Urol 2008; 179 : 952-955; discussion 955.

-20 Otrock ZK, Oghlakian GO, Salamoun MM, Haddad M, Bizri AR: Incidence of urinary tract infection following transrectal ultrasound guided prostate biopsy at a tertiarycare medical center in Lebanon. Infect Control Hosp Epidemiol 2004;25:873-877.

-21 Siriboon S, Tiengrim S, Taweemongkongsup T, Thamlikitkul V, Chayakulkeeree M: Prevalence of antibiotic resistance in fecal flora of patients undergoing transrectal ultrasoundguided prostate biopsy in Thailand. Urol Int 2012;88:187-193.

22 Gil-Vernet Sedo JM, Alvarez-Vijande García $\mathrm{R}$ : Effect of intrarectal povidone-iodine in the incidence of infectious complications after transrectal prostatic biopsy. Arch Esp Urol 2012;65:463-466.

-23 Abughosh Z, Margolick J, Goldenberg SL, Taylor SA, Afshar K, Bell R, et al: A prospective randomized trial of povidone-iodine pro- phylactic cleansing of the rectum before transrectal ultrasound guided prostate biopsy. J Urol 2013;189:1326-1331.

24 Chan ES, Lo K, Ng C, Hou S, Yip SK: Randomized controlled trial of antibiotic prophylaxis regimens for transrectal ultrasoundguided prostate biopsy. Chin Med J (Engl) 2012;125:2432-2435.

25 Madden T, Doble A, Aliyu SH, Neal DE: Infective complications after transrectal ultrasound-guided prostate biopsy following a new protocol for antibiotic prophylaxis aimed at reducing hospital-acquired infections. BJU Int 2011;108:1597-1602

26 Pace G, Carmignani L, Marenghi C, Mombelli G, Bozzini G: Cephalosporins periprostatic injection: are really effective on infections following prostate biopsy? Int Urol Nephrol 2012;44:1065-1070.

27 Gottesman T, Yossepowich O, HarariSchwartz O, Tsivian A, Idler J, Dan M: The value of rectal cultures in treatment of sepsis following post-transrectal ultrasound-guided prostate biopsy. Urol Int 2015;95:177-182.

28 Duplessis CA, Bavaro M, Simons MP, Marguet C, Santomauro M, Auge B, et al: Rectal cultures before transrectal ultrasound-guided prostate biopsy reduce post-prostatic biopsy infection rates. Urology 2012;79:556-561.

29 Taylor AK, Zembower TR, Nadler RB, Scheetz $\mathrm{MH}$, Cashy JP, Bowen D, et al: Targeted antimicrobial prophylaxis using rectal swab cultures in men undergoing transrectal ultrasound guided prostate biopsy is associated with reduced incidence of postoperative infectious complications and cost of care. J Urol 2012;187:1275-1279.
30 Liss MA, Kim W, Moskowitz D, Szabo RJ: Comparative effectiveness of targeted vs empirical antibiotic prophylaxis to prevent sepsis from transrectal prostate biopsy: a retrospective analysis. J Urol 2015;194:397-402.

- 31 Hanna MY, Tremlett C, Josan G, Eltom A, Mills R, Rochester M, Livermore DM: Prevalence of ciprofloxacin-resistant Enterobacteriaceae in the intestinal flora of patients undergoing transrectal prostate biopsy in Norwich, UK. BJU Int 2015;116:131-134.

32 Taylor S, Margolick J, Abughosh Z, Goldenberg SL, Lange D, Bowie WR, et al: Ciprofloxacin resistance in the faecal carriage of patients undergoing transrectal ultrasound guided prostate biopsy. BJU Int 2013;111:946-953.

33 Schellhammer PF, Schaeffer A: Infection associated with transrectal ultrasound guided biopsies of the prostate. PCRI Insights 2011; 14:24-25.

34 Chang Q, Wang W, Regev-Yochay G, Lipsitch M, Hanage WP: Antibiotics in agriculture and the risk to human health: how worried should we be? Evol Appl 2015;8:240-247.

35 Steensels D, Slabbaert K, De Wever L, Vermeersch P, Van Poppel H, Verhaegen J: Fluoroquinolone-resistant $\mathrm{E}$. coli in intestinal flora of patients undergoing transrectal ultrasound-guided prostate biopsy - should we reassess our practices for antibiotic prophylaxis? Clin Microbiol Infect 2012;18:575-581.

36 Cussans A, Somani BK, Basarab A, Dudderidge TJ: The role of targeted prophylactic antimicrobial therapy before transrectal ultrasonography-guided prostate biopsy in reducing infection rates: a systematic review. BJU Int 2016;117:725-731. 